\title{
NOUVELL
}

\section{Les connexions neuronales en pleine lumière}

L'organisation des réseaux inhibiteurs dans le néocortex

Élodie Fino
Équipe dynamique et physiopathologie des réseaux neuronaux, centre interdisciplinaire de recherche en biologie (CIRB), CNRS UMR7241 Inserm U1050, Collège de France, 75005 Paris, France.

elodie.fino@gmail.com
> D’un point de vue phylogénétique, le cortex est la structure cérébrale la plus évoluée. II constitue la base neuronale de nombreuses fonctions allant de fonctions sensorielles ou motrices jusqu'à des fonctions cognitives élaborées. Le fonctionnement du cortex repose sur la mise en jeu de réseaux neuronaux complexes. La connaissance exacte de l'organisation anatomique et fonctionnelle de ces réseaux est donc déterminante pour une meilleure compréhension des fonctions corticales. Cependant, cette tâche est rendue très délicate en raison du nombre et de la variété des neurones qui composent ces réseaux et parce qu'il est nécessaire de comprendre comment chaque type cellulaire s'intègre au cœur des circuits corticaux.

\section{Connaître la structure des circuits} corticaux pour comprendre leur fonction Les premières études des réseaux corticaux se sont focalisées sur les connexions excitatrices existant entre les cellules pyramidales, les neurones principaux du cortex. Les cellules pyramidales sont les éléments clés du traitement de l'information. Cependant, le cortex est également constitué d'interneurones inhibiteurs GABAergiques qui représentent environ $20 \%$ des neurones corticaux. Ces interneurones jouent un rôle déterminant puisqu'ils exercent un effet inhibiteur important sur les cellules pyramidales en contrôlant leur activité de décharge. Leur rôle est dramatiquement illustré dans des pathologies sévères, telles que l'épilepsie ou la schizophrénie, puisqu'un dysfonctionnement de l'activité inhibitrice de ces neurones serait à l'origine des symptômes observés [1, 2]. L'étude des réseaux GABAergiques inhibiteurs est donc essentielle à la compréhension du traitement des informations corticales. Il existe différents types d'interneurones corticaux qui établissent des contacts spécifiques avec différentes parties des cellules pyramidales, allouant à chacun d'entre eux une spécificité dans la régulation de l'activité de ces cellules et donc du réseau cortical [3]. Il est donc nécessaire d'étudier indépendamment chaque type d'interneurones. Dans cette Nouvelle, nous nous intéresserons aux interneurones GABAergiques qui expriment la somatostatine et qui représentent $30 \%$ des interneurones GABAergiques corticaux. Ils ciblent préférentiellement les dendrites des cellules pyramidales, contrôlant ainsi l'excitabilité dendritique et l'intégration des entrées synaptiques $[4,5]$. Mais pour comprendre pleinement le rôle de ces interneurones dans la modulation des réseaux fonctionnels excitateurs, il est également nécessaire de caractériser l'organisation anatomique et fonctionnelle des réseaux inhibiteurs. Ceci nécessite de quantifier la proportion des afférences inhibitrices, mais également de déterminer si elles ciblent de manière très spécifique les réseaux fonctionnels excitateurs ou si, au contraire, elles exercent un contrôle global.

\section{Comment étudier la connectivité neuronale?}

La majorité des études de connectivité neuronale sont réalisées au moyen d'enregistrements électrophysiologiques doubles en patch-clamp qui permettent, à l'aide d'électrodes, de tester les connexions entre les neurones présynaptiques et postsynaptiques. Cependant, cette approche ne permet pas d'avoir une vue d'ensemble du réseau et de sa connectivité en raison du faible nombre de cellules enregistrées simultanément. Pour résoudre ce problème, il est nécessaire d'utiliser une technique qui permet de tester rapidement plusieurs connexions dans les mêmes conditions expérimentales tout en gardant une résolution cellulaire. Une combinaison de techniques dynamiques associant la photolyse de glutamate, la microscopie biphotonique et le multi patch-clamp permet maintenant la dissection anatomo-fonctionnelle des microcircuits neuronaux [6]. Cette combinaison de techniques permet de définir simultanément les connexions fonctionnelles entre plusieurs dizaines de cellules avec une résolution spatiale extrêmement fine (de l'ordre du micromètre) et, ainsi, de tester à une large échelle l'organisation des réseaux inhibiteurs au sein d'un microcircuit cortical.

\section{La photolyse biphotonique de glutamate : un outil pour révéler les connexions neuronales}

La photolyse biphotonique de glutamate permet d'activer les neurones optiquement, grâce à un laser, de manière extrêmement ciblée. Le principe consiste à libérer du glutamate à proximité immédiate d'un neurone, pour qu'il se fixe sur les récepteurs du neurone ciblé et 

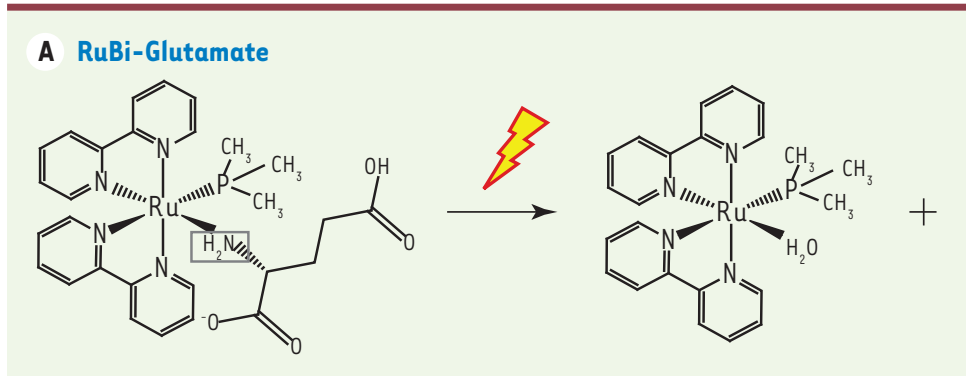

Glutamate
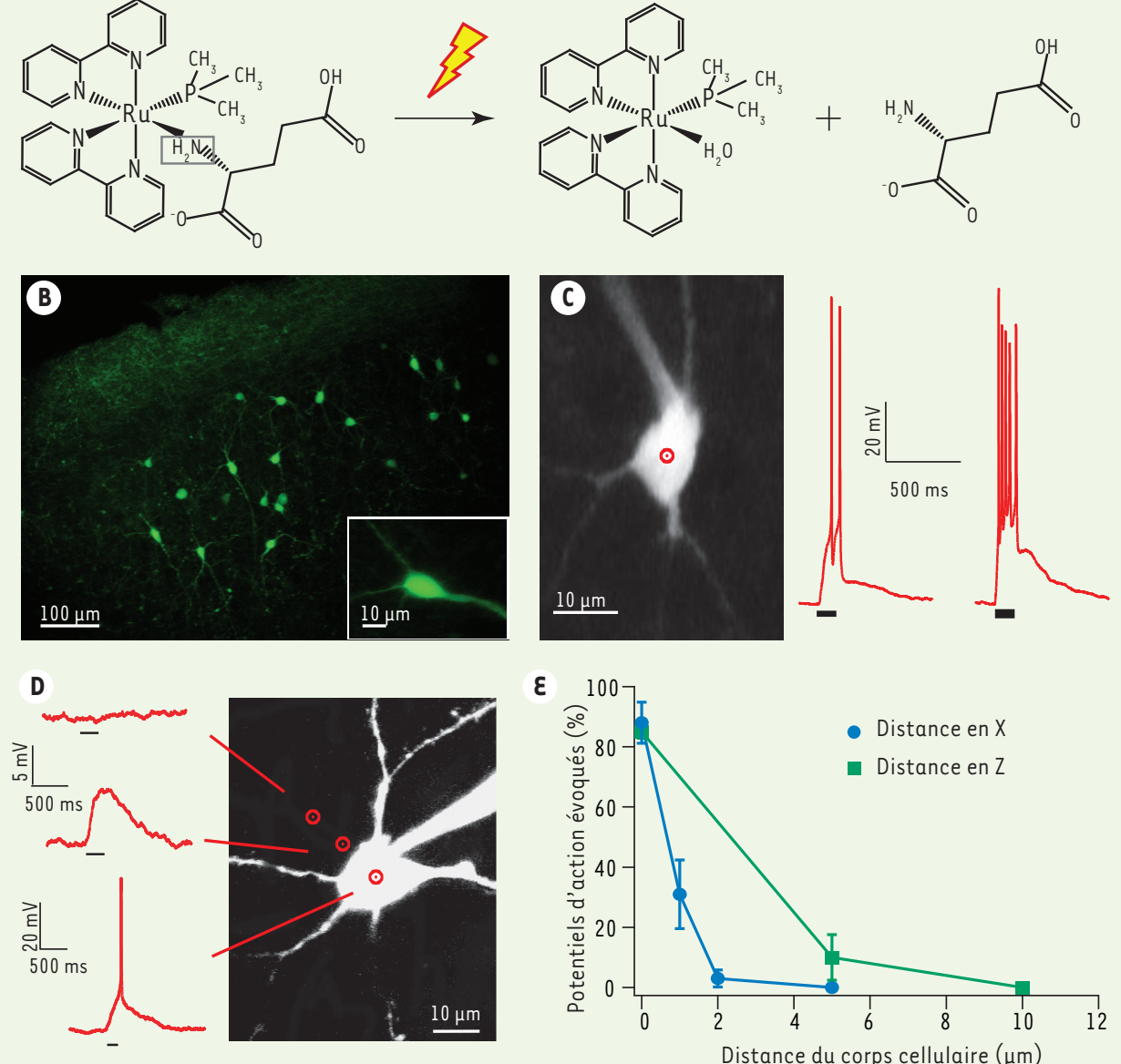

Figure 1. Le RuBi-Glutamate : une nouvelle molécule optimisée pour la photolyse biphotonique. A. Lorsque le RuBi-Glutamate (gauche) est illuminé par le laser, la liaison entre la cage et le glutamate est rompue et le glutamate est libéré (droite). B. Photographie en microscopie biphotonique d'une tranche de cortex de souris dont les interneurones à somatostatine expriment la GFP. C. Exemple d'un neurone enregistré en patch-clamp sur lequel on cible le laser (photographie de gauche). La photolyse biphotonique de RuBi-Glutamate (indiquée par les traits en gras sur le graphe) entraîne l'activation du neurone et le déclenchement de potentiels d'action (courbes rouges). $D-\varepsilon$. Le RuBi-Glutamate permet d'activer les neurones avec une excellente résolution spatiale car le déplacement du laser de quelques micromètres en dehors du corps cellulaire annule les potentiels d'action évoqués (repris de $[7,8]$ ).

entraîne son activation par le déclenchement de potentiels d'action. Cette technique utilise un composé de glutamate "cagé », où le glutamate est couplé à une molécule (appelée molécule cage) le rendant ainsi biologiquement inactif et donc incapable de se fixer à ses récepteurs (Figure IA). Le RuBi-Glutamate [7], un composé développé récemment, s'avère la plus efficace des molécules utilisées jusqu'ici. $\varepsilon$ n réponse à l'illumination du RuBiGlutamate par le laser, le glutamate

est libéré et peut désormais activer le neurone ciblé (Figure IA). Ainsi, il est possible de cibler et d'activer un type neuronal précis dans des tranches de cerveaux de souris dans lesquelles une sous-population neuronale exprime la green-fluorescent protein (GFP). L'enregistrement simultané en patch-clamp du neurone ciblé permet de voir les potentiels d'action déclenchés par la photolyse du glutamate (Figure IC). De plus, l'illumination à des longueurs d'onde élevées (possible uniquement en microscopie biphotonique) permet d'activer les neurones avec une très bonne résolution spatiale et temporelle. Ainsi, si l'on déplace le laser de quelques micromètres en dehors du neurone ciblé, le glutamate ne pourra plus évoquer de potentiels d'action car il sera libéré «trop loin» (Figure IDE). Cette technique permet donc d'activer les neurones avec une très grande précision, à la fois spatiale et temporelle. De plus, le déplacement rapide (de l'ordre de quelques millisecondes) du laser d'un neurone à l'autre permet d'analyser un grand nombre de connexions neuronales et ce très rapidement.

\section{Identification de réseaux inhibiteurs denses dans les circuits corticaux}

$\varepsilon n$ utilisant cette combinaison de techniques, nous avons étudié l'organisation des réseaux inhibiteurs que forment les interneurones GABAergiques à somatostatine contactant les cellules pyramidales dans le cortex frontal (dans les couches superficielles 2/3) [8]. Ainsi, les interneurones localisés dans les tranches de cortex frontal de souris exprimant la somatostatine-GFP (Figure IB) ont été activés séquentiellement par photolyse biphotonique du RuBi-Glutamate et les réponses évoquées dans deux ou trois cellules pyramidales ont été enregistrées simultanément en multi patch-clamp (Figure 2A). Afin de prendre en compte un maximum d'interneurones présents dans le réseau étudié, nous avons choisi un large champ d'investigation de 600 $x 800 \mu \mathrm{m}$. La microscopie biphotonique permet de stimuler les interneurones séquentiellement dans différents plans 

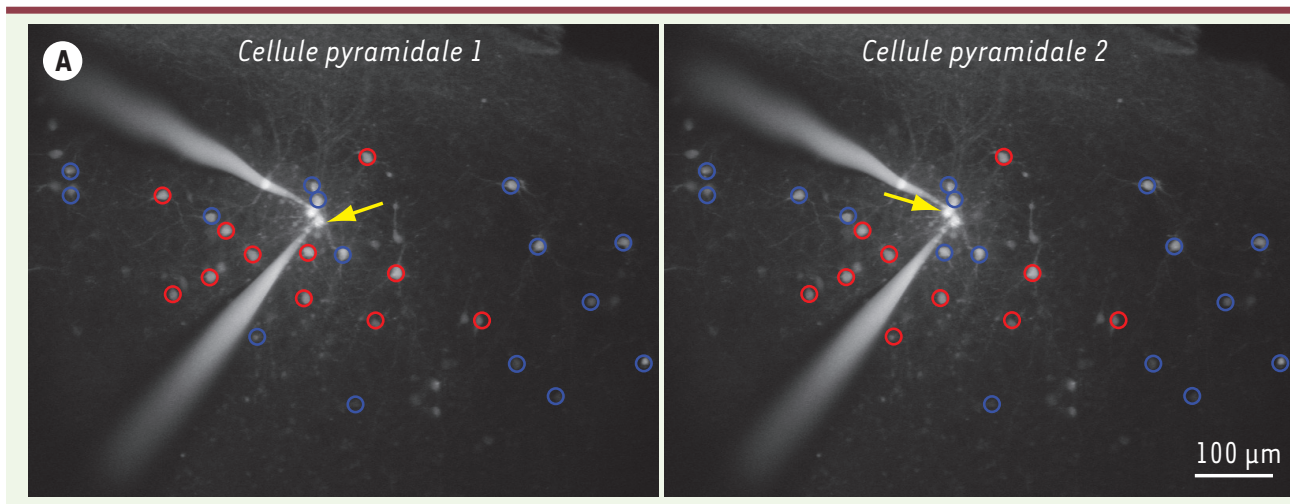

B

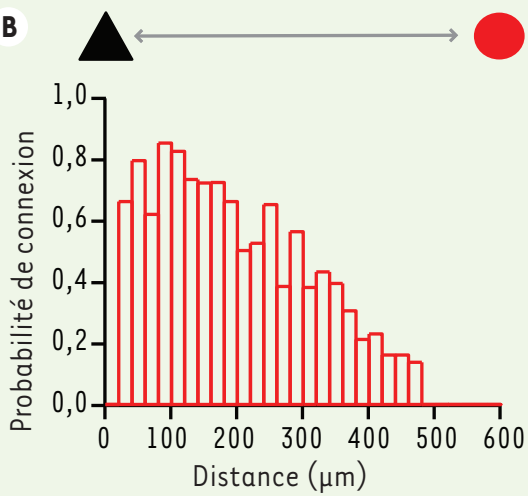

C

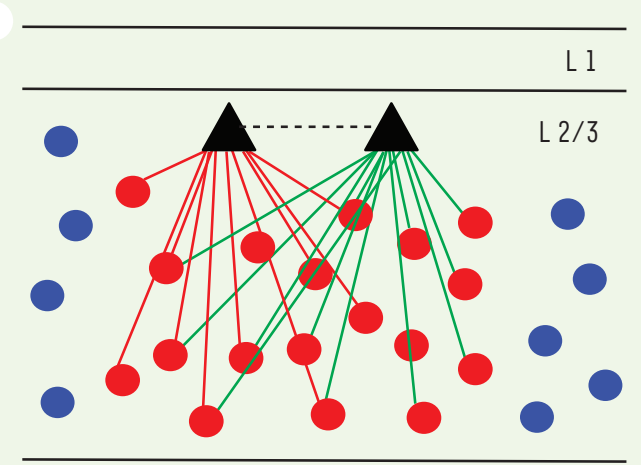

Figure 2. Cartographie des connexions inhibitrices dans la couche $2 / 3$ du cortex frontal. A. Photographies en microscopie biphotonique montrant l'ensemble des interneurones GFP stimulés par photolyse biphotonique du RuBiGlutamate avec un enregistrement parallèle des réponses évoquées dans deux cellules pyramidales. Les cercles rouges indiquent les interneurones connectés aux cellules pyramidales et les bleus les interneurones non connectés (cellule 1 à gauche, cellule 2 à droite). B. La probabilité de recevoir des connexions inhibitrices pour une cellule pyramidale est très importante localement et décroît en fonction de la distance. $C$. Représentation schématique de la densité des connexions inhibitrices sur les cellules pyramidales, que celles-ci soient connectées entre elles ou non (repris de [8]).

axiaux et sur une profondeur de l'ordre de $100 \mu \mathrm{m}$. Ainsi, l'ensemble des interneurones présents autour des cellules pyramidales et appartenant à un microcircuit local ont pu être testés, soit de 10 à 45 interneurones dans le même champ cortical. Cette méthodologie permet d'analyser un grand nombre de connexions dans les mêmes conditions expérimentales (Figure 2A).

Ces expériences ont permis de construire des cartographies de réseaux inhibiteurs (Figure 2A). Ces cartographies ont montré que les cellules pyramidales reçoivent une innervation massive des interneurones à somatostatine puisque, localement, 70 à $80 \%$ des interneurones sont en contact avec les cellules pyramidales enregistrées. Ce pourcentage est deux à trois fois plus élevé que celui qui est observé avec des doubles ou triples enregistrements en patch-clamp [9]. Cette différence s'explique probablement par le nombre beaucoup plus élevé de connexions inhibitrices qui ont pu être testées, mais également par le fait que la microscopie biphotonique permet d'étudier les cellules localisées profondément dans la tranche de cerveau, optimisant les chances de préserver les connexions intactes. II existe également une forte corrélation entre la connectivité et la distance entre les interneurones et les cellules pyramidales (Figure 2B). Ainsi, une cellule pyramidale proche des interneurones aura plus de chances d'être en contact avec ces interneurones qu'une cellule plus éloignée. Cependant, même pour des distances supérieures à $300 \mu \mathrm{m}$, $40 \%$ des interneurones sont connectés aux cellules pyramidales. Ces expériences révèlent donc que la densité des réseaux inhibiteurs locaux est beaucoup plus importante que ce qui avait été décrit précédemment.

D'autre part, grâce à l'enregistrement électrophysiologique simultané de plusieurs cellules pyramidales voisines, nous avons pu comparer les cartographies de réseaux inhibiteurs pour des cellules pyramidales connectées appartenant à un même réseau fonctionnel, ou des cellules pyramidales non connectées appartenant à des réseaux fonctionnels distincts. La Figure $2 A$ illustre un exemple de cartographies inhibitrices pour deux cellules pyramidales voisines non connectées. On y observe la similitude des réseaux inhibiteurs pour ces deux cellules pyramidales voisines. De façon surprenante, nous avons observé un même degré de similitude que les cellules pyramidales soient connectées ou non (Figure 2C). Ces résultats démontrent que la spécificité de connectivité des entrées inhibitrices est indépendante de la connectivité entre les cellules pyramidales.

L'organisation anatomofonctionnelle des réseaux inhibiteurs ainsi cartographiés révèle une densité des connexions inhibitrices beaucoup plus importante que ce qui avait été observé précédemment, renforçant l'importance fonctionnelle de l'inhibition dans les circuits corticaux. De plus, de manière 
surprenante, la spécificité de connexion de ces réseaux inhibiteurs semble indépendante des microcircuits excitateurs locaux. Le rôle des réseaux inhibiteurs n'apparaît donc pas comme restreint au contrôle précis d'un réseau fonctionnel mais plutôt comme un contrôle global local de la balance excitation/ inhibition et une modulation de la sortie fonctionnelle des circuits corticaux. $\diamond$ Dense inhibitory neuronal networks revealed by two-photon photoactivation of RuBi-Glutamate

\section{CONFLIT D'INTÉRÊTS}

L'auteur déclare n'avoir aucun conflit d'intérêts concernant les données publiées dans cet article.

\section{RéFÉRENCES}

1. Cossart R, Bernard C, Ben-Ari Y. Multiple facets of GABAergic neurons and synapses: multiple fates of GABA signalling in epilepsies. Trends Neurosci 2005 ; $28: 108-15$.

2. Lewis DA, Hashimoto T, Volk DW. Cortical inhibitory neurons and schizophrenia. Nat Rev Neurosci 2005 ; $6: 312-24$

3. Markram H, Toledo-Rodriguez M, Wang Y, et al. Interneurons of the neocortical inhibitory system. Nat Rev Neurosci 2004 ; 5 : 793-807.

\section{NOUVELL\&}

\section{Le Yin et le Yang de la sénescence}

Est-il possible de vieillir sans développer de cancer?

Matthieu Lacroix, Laetitia K. Linares, Laurent Le Cam
4. Silberberg G, Markram H. Disynaptic inhibition between neocortical pyramidal cells mediated by Martinotti cells. Neuron 2007 ; 53 : 735-46.

5. Murayama M, Pâerez-Garci $\varepsilon$, Nevian T, et al. Dendritic encoding of sensory stimuli controlled by deep cortical interneurons. Nature 2009 ; 457 : 1137-41.

6. Nikolenko V, Poskanzer KE, Yuste R. Two-photon photostimulation and imaging of neural circuits. Nat Methods $2007 ; 4$ : 943-50.

7. Fino $\varepsilon$, Araya R, Peterka DS, et al. RuBi-Glutamate: Two-photon and visible-light photoactivation of neurons and dendritic spines. Front Neural Circuits $2009 ; 3: 2$.

8. Fino $\varepsilon$, Yuste, R. Dense inhibitory connectivity in neocortex. Neuron 2011 ; 69 : 1188-203.

9. Thomson AM, Lamy C. Functional maps of neocortical local circuitry. Front Neurosci 2007 ; 1 : 19-42.
Institut de recherche en cancérologie de Montpellier, Inserm U896, centre de lutte contre le cancer Paul Lamarque Val d'Aurelle, Montpellier, 34298, France.

laurent.lecam@inserm.fr

de tumeur : les protéines $\mathrm{pl} 6^{\operatorname{lnk} 4 a}$ et ARF (alternative reading frame, $\mathrm{pl} 4^{\mathrm{Arf}}$ chez I'homme ou pl9Arf chez la souris). Ce locus constitue un point de convergence de nombreux signaux de stress conduisant in fine à la modulation des voies impliquant les suppresseurs de tumeur $\mathrm{Rb}$ et $\mathrm{p} 53$ [3]. Ces derniers sont des régulateurs clé de la sénescence, et les voies qu'ils empruntent représentent l'archétype des voies des suppresseurs de tumeurs. De fait, ces voies de signalisation sont inactivées, à un niveau ou à un autre, dans pratiquement toutes les tumeurs.

Cette stratégie originale a permis à Baker et al. [1] de montrer que la destruction des cellules sénescentes exprimant pl6 lnk4a (et donc le gène suicide) améliorait significativement certains symptômes associés à un vieillissement prématuré dans un modèle murin progéroïde basé sur l'expression d'un allèle hypomorphe de la kinase BubRl (Bub$\mathrm{RI}^{\text {Hyp/Hyp}}$ ) [4]. Ces travaux font écho à de précédentes études démontrant 